\title{
Modulation of mismatch negativity by stimulus deviance and modality of attention
}

\author{
Bernhard W. Müller, Christiane Achenbach, Robert D. Oades, Stefan Bender and Ulrich Schall ${ }^{1}$
}

2002 NeuroReport, 13, 1317-1320

\begin{abstract}
Clinic for Psychiatry and Psychotherapy, and Clinic for Child and Adolescent Psychiatry and Psychotherapy, University of Essen, Virchowstrasse174, 45147 Essen, Germany.

(1) Centre for Mental Health Studies, University of Newcastle, Australia
\end{abstract}

\begin{abstract}
We studied the effect of attention on the processing of auditory sensory inputs by means of the mismatch negativity (MMN) potential, which can be derived from event-related EEG. A series of frequent standard and rare deviant auditory stimuli were presented to 20 healthy subjects in two recording sessions about five weeks apart. Deviant stimuli were either low or highly deviant as compared to the standard stimulus. While MMN was recorded, subjects were performing a visual and, subsequently, an auditory discrimination task. Directing attention towards the auditory task was associated with increased MMN amplitude only in response to low deviant stimuli and only in the first recording session. No change of MMN amplitude was found when directing attention towards the visual task or when MMN was recorded in response to highly deviant auditory stimuli. The latter may trigger an involuntary switch of attention, thereby overwriting the effect of task-directed attention. Conversely, the effects of attention on the processing of low deviant stimuli appear to be fragile and diminish with increasing automaticity of task execution.
\end{abstract}

Key words: Attention; Event-related potentials; P300; Evoked potentials; auditory; Mismatch negativity; Novelty processing; Repeated measurement

Acknowledgements: We would like to thank Professors M. Gastpar and C. Eggers for substantial support, R. Franzka, R. Haverkorn, R. Windelschmitt, and D. Zerbin for assisting in data acquisition, and P. Johnston and Dr. M. Cohen for valuable feedback on our manuscript. Dr. Schall was supported by NSW Health infrastructure funding.

\section{INTRODUCTION}

The analysis of new events occurring outside the focus of attention is a fundamental ability of human information processing. Most sensory events which occur within every day activities have to be ignored while others have to be assigned for further analysis according to their relevance for ongoing information processing [1]. A major contribution to the assessment of cortical information processing to unexpected events within the auditory modality has been provided within research on event-related potentials (ERP). While regular events elicit a cortical event related potential which mainly consists of the N100 component and the P200 component, rare deviant events lead to an additional (electrically negative) ERP component at about 100-250 ms after stimulus onset. This component has been named mismatch negativity, indicating that it is elicited by events which impose some kind of deviance from a previously established con-text of standard stimuli [2]. Technically, the amplitude of the MMN can be derived by subtracting the event-related potential to the standard stimulus from the event-related potential to the deviant stimulus. Most studies on MMN have focussed on auditory information processing, although recent reports provide evidence of MMN-like responses to somatosensory and visual deviant processing [3,4]. The auditory MMN has been recorded in response to frequency, duration and intensity deviance as well as to feature variations that occur within complex sound patterns [5-7].

Näätänen et al. suggested that MMN (elicited by acoustic stimuli) is a measure of auditory 
sensory memory, thus reflecting an attentionindependent and automatic response (i.e. without conscious perception) in the primary and secondary auditory cortex $[2,8]$. However, several studies have demonstrated modulation of auditory sensory memory by attention [9-12]. Woods et al. [9] and Oades et al. [10] found evidence for enhanced MMN amplitudes whilst attention is directed towards a concurrently performed simple discrimination task. Conversely, DittmannBalcar et al. [11] reported an attenuation of MMN amplitude with increasing difficulty of the concurrently performed auditory discrimination task. Wolldorf et al. [12] further confirmed an effect of attention on MMN when employing a dichotic listening task by assessing the magneto-encephalographic equivalent of the MMN. These findings, as well as neuroimaging studies reporting intramodal upregulation of the superior temporal gyrus under active attention conditions when compared to a passive listening task, support the notion of partially over-lapping neural networks that are concurrently serving attention and sensory memory function [13].

In contrast to these results which assessed MMN with low to moderate deviance contrasts, Woods et al. [9] reported no effects of attention on MMN amplitude when using high levels of deviance contrast. Hence, dependent on stimulus salience, distinct neural processes appear to underlie MMN attention modulation. For instance in the case of higher deviance contrasts, an additional orienting response is more likely to be elicited, thus promoting active stimulus evaluation [1]. Further evidence for distinct modes of MMN generation derives from clinical studies reporting differential $\mathrm{MMN}$ attenuation across different levels of stimulus salience. For instance, MMN amplitude reduction in the auditory domain has been consistently reported in schizophrenia with low deviance contrasts (i.e. duration decrement) [14] while no $\mathrm{MMN}$ attenuation was found with high deviance contrast [7].

The current study aims to assess attention interference with auditory sensory memory processing of low versus high levels of stimulus salience in the context of directed attention towards the auditory or visual domain in a repeated measurement design. It is hypothesised that only low levels of stimulus deviance are associated with a modality of attention-dependent modulation of MMN amplitude [9], and that this effect diminishes with increasing automaticity of the distractor task due to a reduction of attention demands and resulting down-regulation of the sensory association cortices [15].

\section{MATERIAL AND MEthODS}

Subjects and stimuli: We tested 20 righthanded healthy volunteers (university students with a mean (7s.d.) age of $25.8 \pm 3.7$ years) twice within $37.3 \pm 9.8$ days. No subject had previous experience with ERP research. Stimuli were generated and presented by using the NeuroStim software package (Neuroscan Inc.). Four types of auditory stimuli were presented binaurally at $76 \mathrm{~dB}$ (SPL) via headphones with a stimulus onset asynchronicity (SOA) of $1 \mathrm{~s}$ : (1) $1000 \mathrm{~Hz}$ with $80 \mathrm{~ms}$ duration including $10 \mathrm{~ms}$ rise/ fall time (standard tone, $p=0.82$ ), (2) 1000 $\mathrm{Hz}$ with $40 \mathrm{~ms}$ duration including $5 \mathrm{~ms}$ rise/ fall time (duration deviant tone, $p=0.06$ ), (3) $500 \mathrm{~Hz}$ with $80 \mathrm{~ms}$ duration including $10 \mathrm{~ms}$ rise/fall time (frequency deviant tone, $\mathrm{p}=0.06$ ), and (4) non repeating and non-identifiable computergenerated complex sound patterns with $80 \mathrm{~ms}$ duration including $10 \mathrm{~ms}$ rise/fall time (novelty deviant stimuli, $\mathrm{p}=0.06$ ). In addition, red and green circles (visual angle of 2.81) were concurrently presented on a computer screen in front of the subject.

Task description: Subjects were comfort-ably seated in an electrically shielded room with dimmed illumination. In the visual-attend condition, 1200 auditory stimuli were presented in a series of four consecutive recording blocks of 300 stimuli each while subjects performed a visual discrimination task on the circles presented with a randomised probability of $50 \%$ and an inter-stimulus interval (ISI) of $1.1 \mathrm{~s}$ by pressing a button (Stimpad, Neuro-scan Inc.) in order to remove the red circle from the screen. Thereafter, subjects performed an auditory discrimination task on another 1200 auditory stimuli (also divided into four recording blocks) by responding to the frequency deviant tones with a button press while the red and green 
circles were alternated with an ISI of $1.1 \mathrm{~s}$ with $1.5 \mathrm{~s}$ presentation duration (range 1.0-2.0) and not requiring a response. Responding with the right or left index finger was alternated between consecutive stimulus blocks in both conditions, respectively.

ERP recordings and analysis: ERP data were recorded from 32 leads (extended international 10-20 system; Electrocap Inc.) using a digital band pass filter of $0.1-30 \mathrm{~Hz}$ (Scan 3.0, Neuroscan Inc.) with linked earlobe reference. Trials exceeding $750 \mathrm{mV}$ in horizontal or vertical electro-oculogram (EOG) or at frontal leads (Fp1, Fp2) were discarded from analysis. A correction for eye-blink artifacts was performed in two subjects due to a high amount of eye blink contamination [16]. ERPs were calculated for the standard tone, the duration deviant tone, and the novel stimuli in the auditory and the visual attend conditions, respectively, over 900 ms poststimulus onset intervals relative to a baseline of $100 \mathrm{~ms}$ prior to stimulus onset. Mean MMN amplitudes were calculated at $\mathrm{Fz}$ for five consecutive $40 \mathrm{~ms}$ intervals (from 60 to 260 $\mathrm{ms})$ as difference waves: duration deviantstandard stimuli and novel-standard stimuli, respectively.

\section{RESULTS}

Averaged ERPs were calculated from 664 to 704 responses to standard and from 46 to 54 responses to deviant stimuli for both conditions per subjects. Eye-blink contamination did not vary across type of deviance, attention conditions, and test sessions. In the auditory discrimination task, mean response time was $437 \pm 55$ ms vs. $436 \pm 65 \mathrm{~ms}$ at re-test. In the visual task mean response time was $438 \pm 85 \mathrm{~ms}$ vs. $428 \pm 72 \mathrm{~ms}$ at re-test. There were no significant differences in reaction time with regard to test sessions and modality. Overall performance between recording sessions improved, particularly errors of commission (or false alarms) in response to novel stimuli significantly decreased from 1.271 .0 initially to 0.5570 .8 at re-test $(t=2.6, d f=19, p<0.02)$.

Attention modulation effects on MMN amplitude were tested with a full-factorial multivariate ANOVA model including the factors (1) modality of Attention (visual/auditory) by (2) type of mismatch (duration/novel) by (3) sessions (test/re-test) by (4) MMN sub-components (five consecutive $40 \mathrm{~ms}$ intervals from 60 to $240 \mathrm{~ms}$ poststimulus onset). MMN amplitude significantly differed across intervals $(F(4,16)=34.0$; $p<0.001)$ with larger MMN amplitudes elicited by novel deviance $(F(1,19)=7.2 ; p<0.02$; Fig.1). The significant interaction of $M M N$ subcomponents - type of mismatch $(F(4,16)=$ 9.9; $p<.001)$ indicated an earlier peak maximum for MMN amplitudes elicited by novel compared to duration deviance, thus also partially reflecting a contribution of a $\mathrm{P} 3 \mathrm{a}$ response. The effect of modality of attention was confirmed in a three-way interaction with type of mismatch and MMN sub-components $(F(4,16)=4.6 ; p=0.01)$.

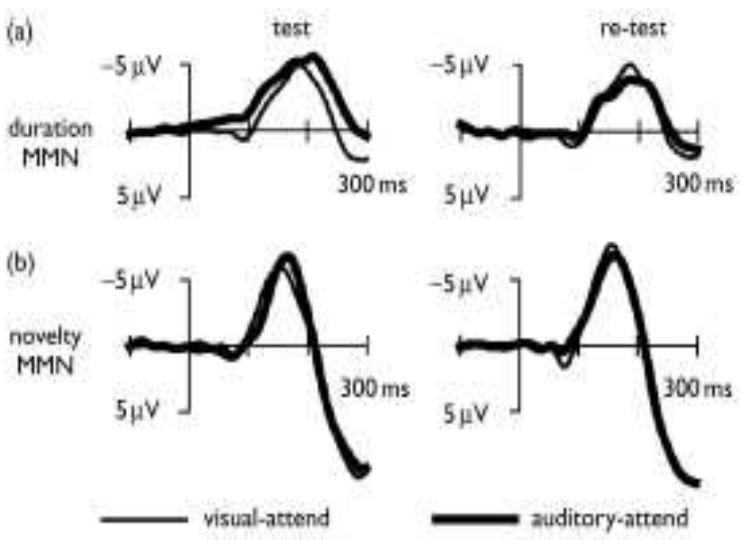

Fig.1. Repeatedly recorded event-related potentials at the $\mathrm{Fz}$ electrode (test/re-test interval of 37 days) of auditory mismatch negativity (MMN) when concurrently performing an auditory or visual discrimination task (negative up). MMN was calculated by subtracting event-related potentials in response to frequent non-target auditory stimuli from those to rare non-target deviant auditory stimuli. Two types of MMN were assessed: (a) in response to low deviance contrast (duration decrement stimuli) and (b) high deviance contrast (complex novel sounds). MMN modulation by directing attention was only found with low deviance contrast in the first test session

The attention effect was then tested separately for type of mismatch and for MMN sub-components with significant MMN. 
Significant MMN $(p<0.05)$ was found within an interval of 100-260 ms for duration mismatch and $100-220 \mathrm{~ms}$ for novelty mismatch. Within these significant intervals, mean amplitudes for duration MMN were calculated with -3.94 $\pm 1.6 \mathrm{mV}$ vs. $-2.43 \pm 1.62 \mathrm{mV}$ at re-test when performing the auditory discrimination task on the frequency deviant stimuli. When performing the visual distraction task, the mean MMN amplitude was_2.53 $\pm 1 / 85 \mu \mathrm{V}$ vs. $2.28 \pm 1.42 \mu \mathrm{V}$ at re-test. Statistical analysis confirmed a larger MMN amplitude when performing the auditory discrimination task vs. the visual task $(F(1,19)=6.9 ; p<0.02)$. The statistical trend of a two-way interaction of modality of attention sessions $(F(1,19)=4.0$; $p<0.06)$ was confirmed as a significant decrease of MMN amplitude at re-test when perform-ing the auditory discrimination task $(F(1,19) \quad=7.8 ; \quad p=0.01 ; \quad F i g . \quad 2)$. The corresponding $\mathrm{MMN}$ amplitude measures for novelty-elicited MMN were $-3.58 \pm 4.03 \mu \mathrm{V}$ vs. $-3.87 \pm 3.25 \mu \mathrm{V}$ at re-test when performing the auditory discrimination task and $-3.47 \pm 2 / 91$ $\mu \mathrm{V}$ vs $-4.24 \pm 2.89 \mu \mathrm{V}$ at re-test when performing the visual task. No modality of attention and type of mismatch effects or differences between recording sessions were statistically confirmed.

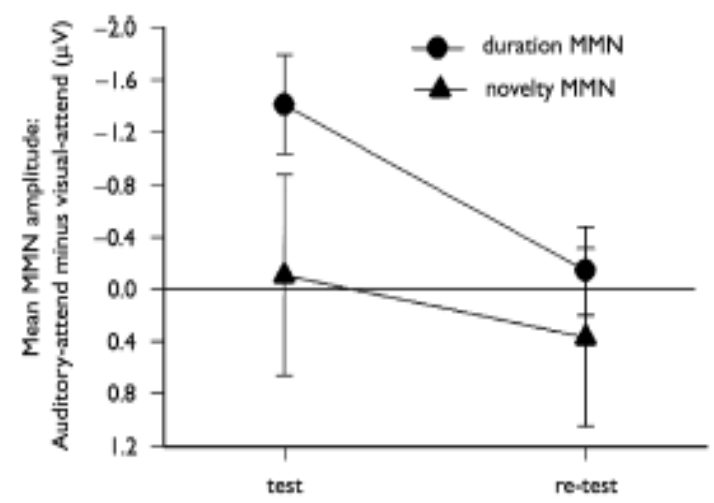

Fig. 2. Modulation of auditory mismatch negativity MMN by directing attention towards a concurrently performed auditory or visual discrimination task. A value of zero indicates no attention modulation effect and negative values indicate larger MMN amplitudes in the auditory attend condition as compared to the visual attend condition. This effect was found to be significant in the initial recording session but not when retested after 37days.

\section{Discussion}

Our results revealed a modality of attentiondependent modulation of MMN amplitude for low but not for high deviant stimulus contrasts [9]. Furthermore, this effect is only initially present and diminishes with repeated recording. This observation has important implications for studies dependent on the reliable assessment of $\mathrm{MMN}$. For instance, the majority of clinical MMN studies have utilised rather simple mismatch conditions (i.e. duration, frequency or intensity) that also control for attention effects by using a visual distraction tasks (e.g. reading a text, watching a video, or performing a visual discrimination task) [14]. Our findings indicate intraindividually stable MMN amplitude recordings over 4-6 weeks when a visual task is concurrently administered.

By contrast, a concurrent task that directs attention towards the auditory modality will affect MMN amplitude measures when using low-deviant stimulus contrasts [9], particularly with increasing concurrent task difficulty [11].

The current data further suggest that this effect diminishes with increasing automaticity of concurrent task execution (as indicated by the improvement of concurrent task performance) due to fewer demands on attention. On the other hand, more complex deviant stimuli, than those used here, are more likely to trigger an orienting response, thus directing attention towards the highly deviant stimulus [17]. This involuntary switch of attention (commonly associated with a P3a response) appears to overwrite the effect of directed attention and increases the probability of an inappropriate response (i.e. errors of commission in the context of our experiment) [1]. There are now converging evidence from ERP and functional brain imaging studies suggesting a fronto-temporal network involved in MMN generation that, at least, involves the posterior part of the superior temporal gyrus (STG) and the inferior frontal gyrus [18-20]. It has been argued that the posterior STG contributes to short-term representations of auditory stimuli [21]. Hence neuronal processing of low-deviance MMN may be more confined to the primary and secondary auditory cortex. Additional 
prefrontal processing appears to be initiated upon violations of the expected sensory input that occurs as a mismatch between shortterm representation (auditory sensory memory) and with increasing degree of deviance contrast [2]. Anatomical evidence for such a mechanism arises from studies on primates confirming cortico-cortical connections from the temporal lobes to frontal areas and via direct projections from subcortical nuclei [22-25].

\section{CONCLUSION}

Our findings support the notion that MMN is mainly an attention-independent measure of automatic auditory information processing. Modality of attention affects MMN amplitude, though this effect appears to be confined to low deviant contrasts under intra-modal attend conditions. Conversely, MMN elicited by high-deviant contrasts appears not to be affected by directing attention owing to a triggering of an involuntary switch of attention towards the novel stimulus, thus over-writing the effects of directed attention. Our re-test results further indicate that attention modulation of $\mathrm{MMN}$ is rather fragile and diminishes with the degree of automaticity that is acquired in the course of task execution.

\section{REFERENCES}

1. Mesulam MM. Brain 121, 1013-1052 (1998).

2. Näätänen R. Behav Brain Sci 13, 201-288 (1990).

3. Wei JH, Chan TC and Luo YJ. Brain Res Bull 57, 221-230 (2002).

4. Astikainen $P$, Ruusuvirta $T$ and Korhonen $T$. Neurosci Lett 298, 222-224

(2001).

5. Alain C, Woods DL and Ogawa KH. NeuroReport 6, 140-144 (1994).
6. Horvath J, Czigler I, Sussman E et al. Brain Res Cogn Brain Res 12, 131-144

(2001).

7. Grzella I, Muller BW, Oades RD et al. J Psychiatry Neurosci 26, 235-246

(2001).

8. Näätänen $\mathrm{R}$, Paavilainen $\mathrm{P}$, Tiitinen $\mathrm{H}$ et al. Psychophysiology 30, 436-450 (1993).

9. Woods $\mathrm{DL}$, Alho $\mathrm{K}$ and Algazi $\mathrm{A}$. Electroencephalogr Clin Neurophysiol 82, 341-355 (1992).

10. Oades RD and Dittmann-Balcar A. Neuroreport 6, 1187-1190 (1995).

11. Dittmann-Balcar A, Thienel $R$ and Schall U. Neuroreport 10, 3749-3753

(1999).

12. Woldorff MG, Hillyard SA, Gallen CC et al. Psychophysiology 35, 283-292 (1998).

13. Hall DA, Haggard MP, Akeroyd MA et al. Hum Brain Mapp 10, 107-119 (2000).

14. Michie PT. Int J Psychophysiol 42, 177-194 (2001).

15. Coull JT. Prog Neurobiol 55, 343-361 (1998).

16. Semlitsch HV, Anderer P, Schuster $P$ et al. Psychophysiology 23, 695-703

(1986).

17. Escera C, Alho K, Winkler I et al. J Cogn Neurosci 10, 590-604 (1998).

18. Müller BW, Jüptner $M$, Jentzen $W$ et al. Neuroimage 10, in press (2002).

19. Dittmann-Balcar $A$, Jüptner $M$, Jentzen $W$ et al. Neurosci Lett 308, 119-122 (2001).

20. Opitz B, Rinne T, Mecklinger A et al. Neuroimage 15, 167-174 (2002).

21. Wise RJS, Scott SK, Blank SC et al. Brain 124, 83-95 (2001).

22. Bignall $K E$ and Imbert $M$. Electroencephalogr Clin Neurophysiol 26, 206215 (1968).

23. Romanski LM and Goldman-Rakic PS. Nature Neurosci 5, 15-16 (2002).

24. Pandya DN. Rev Neurol (Paris) 151, 486494 (1995).

25. Liasis A, Towell A, Alho $K$ et al. Brain Res Cogn Brain Res 11, 227-233 (2001) 